\title{
Recent research in spatial real estate hedonic analysis
}

\section{Antonio Páez}

Published online: 15 November 2009

(C) Springer-Verlag 2009

Keywords Real estate $\cdot$ Hedonic prices $\cdot$ Spatial analysis $\cdot$ Spatial econometrics · Kriging

JEL Classification $\mathrm{R} 31 \cdot \mathrm{C} 01 \cdot \mathrm{C} 21$

\section{Introduction}

The analysis of real estate markets continues to be a prime area for the application and development of spatial analysis techniques. While spatial analytical approaches have become more widespread and accessible over the past decade to researchers and practitioners in a wide variety of disciplines (Páez et al. 2009), it is interesting to note that some early examples of the potential of both spatial econometric and geostatistical techniques include the analysis of property values reported in papers by Can (1992) and Dubin (1992). These early papers are notable because they demonstrated the use of different analytical approaches-spatially autoregressive models in the case of Can, and kriging in the case of Dubin. At the same time, they also identified a series of issues that stand at the cornerstone of spatial hedonic analysis. Of these issues, the identification of spatial autocorrelation and heterogeneity is a key, and these two effects have since been consistently found in other studies and with such frequency that it is no longer reasonable to dismiss them as statistical flukes or nuisances. On the contrary, while a strong economic theory of spatial externalities and segmentation in real estate markets has yet to be produced, a current consensus regarding the interpretation of spatial effects has already

\footnotetext{
A. Páez $(\bowtie)$

Centre for Spatial Analysis/School of Geography and Earth Sciences,

McMaster University, 1280 Main Street West, Hamilton, ON L8S 4K1, Canada

e-mail: paezha@mcmaster.ca
} 
emerged. Thus, neighborhood effects are a commonly accepted interpretation of patterns of similarities observed in property prices above and beyond what can be explained by differences in structural and locational attributes (e.g. Baumont 2009; Chica-Olmo 1995; Pace et al. 1998; Tse 2002). Heterogeneity, on the other hand, is believed to be indicative of geographical segmentation of markets or buyers preferences (e.g. Bitter et al. 2007; Bourassa et al. 2003; Goodman and Thibodeau 2003; Páez et al. 2008b). The literature on spatial real estate hedonic analysis has so far demonstrated the importance of spatially explicit modeling to improve model quality and prediction accuracy. Substantive interpretation lends credence to the use of these methods. And the policy implications of spatial spillovers are increasingly recognized as a sufficient justification for the application of specialized approaches. Research continues to thrive as new substantive problems are identified, and methods are adopted, adapted, or newly developed to address emerging research needs. The papers collected for this thematic issue of Journal of Geographical Systems are a sample of research in an area that continues to attract attention for its methodological potential and practical importance.

The first paper in the issue is by Leonard and Murdoch, and is concerned with the neighborhood effects of foreclosure. As is well known, residential real estate markets were a central element in the financial and economic crisis of 2008. As the housing bubble in these markets came to an end and market prices started to sharply adjust, many homeowners found themselves holding so-called underwater mortgages (mortgages with high Loan-to-Value ratios), and in a situation where the money owed in a mortgage exceeded the market value of the property. With many homeowners also having obtained in the preceding years exotic mortgages that created the illusion of cheap credit early on, unsustainable financial conditions after the burst of the bubble pushed many towards default-since at that point it would have been more expensive to hold on to a property than to walk away from it. There have been multiple media accounts of the geographical concentrations of foreclosure, and doubtless these concentrations can to a large extent be explained by common factors, such as low neighborhood income. Formal statistical research has also been devoted to the question of the neighborhood effects of foreclosure, in order to determine whether the effects of foreclosure tend to spillover and negatively affect nearby properties. The question is of primary interest, because negative effects could spread, pushing otherwise healthy properties into default. At the same time, it is possible that some of the worse effects of widespread foreclosures could be contained by taking a geographically targeted approach for policy interventions.

A challenge when attempting to estimate the neighborhood effects of foreclosure is explained by Harding et al. (2009) in the following way: "If the empirical model does not control for the overall price level, a large number of nearby foreclosures may proxy for an overall decline in home prices and thus a negative coefficient on the measure of foreclosures could actually be an estimate of the local decline in prices and not a true contagion effect." Given abundant previous evidence that property prices tend to be spatially autocorrelated, it can be argued that the empirical model needs to take into account not only the "overall" price level, but also the locally adjusted price level. A spatial approach provides precisely the tools 
to achieve this, by introducing a local moving average that is a function of the price of neighboring properties. Two possibilities emerge that must be considered: first, that the negative coefficient for foreclosures confounds overall and local price levels with other sources of market distress, and thus provides an inflated estimate of the effect of foreclosures per se; and secondly, that parameter estimates are further biased in complex ways if autocorrelation effects are indeed present but are ignored (see Páez et al. 2008a). Leonard and Murdoch demonstrate that the effect of foreclosures follows a distance decay pattern, with the impacts becoming increasingly small with increasing distance. Furthermore, their results show that the coefficient for number of foreclosures is negative and significant, but smaller than would be indicated if neighborhood price effects are not addressed. The effect of foreclosures turns out to be fairly complex, as it consists of a negative direct effect (attached to the presence of foreclosed properties), as well as an indirect propagation effect that makes the prices of properties to covary as a cluster. Thus, in the final analysis, Leonard and Murdoch estimate that the total direct and indirect (neighborhood) hedonic price effect of one additional foreclosed property within 250 feet is $-\$ 1,666$. A model that ignores neighborhood effects, in contrast, produces an inflated estimated effect of $-\$ 2,400$ per one additional foreclosure. Along with recent research by Clauretie and Daneshvary (2009), this paper gives the clearest evidence available so far of the importance of considering the contagion effects of foreclosure in property markets.

The second paper in the issue, by Smith and $\mathrm{Wu}$, is motivated by the need to assess the impact of community development projects such as implemented in the Philadelphia region. The aim of these projects is to revitalize local neighborhoods, and housing prices are used as a summary measure to evaluate the success of an intervention. Most work to date in the spatial analysis of real estate data has been completed from the perspective of cross-sections. Usually, price data are collected over a period of time (one or more quarters or a year), and then analyzed as a co-temporal spatial series. While this practice greatly simplifies the need to work with potential dependencies along the temporal dimension, it imposes the limitation that time shifts cannot be meaningfully analyzed (for example, trying to identify higher resolution temporal effects before and after a project). Moreover, dependencies along the temporal dimension are all given the same weight, when it is reasonable to anticipate that any effects would tend to fade over time. Since transactions, and therefore sale prices are recorded on a daily basis, and at irregular intervals of time, Smith and Wu propose a new modeling approach that is capable of accommodating both spatial and temporal dependencies. The application of the model to a revitalization project in Philadelphia (the People's Emergency Center) is illustrative, and reveals significant if weak levels of temporal autocorrelation. It is important to note that the model is derived for the case of data points that do not coincide in time, which explains why the spatial lag can be estimated by ordinary least squares (see Stetzer 1982). In small sample situations, this may reasonably be expected to hold if the time period is relatively long. In many practical situations that involve larger samples, it is not uncommon to find that several transactions are recorded for a single day. While analytically the situation becomes more complex, 
Smith and $\mathrm{Wu}$ sketch a possible solution to the problem and indicate that research is ongoing on this matter.

A quick survey of the literature suggests that applications of spatial econometric approaches appear to be more numerous in the literature compared to geostatistical approaches involving the use of kriging methodology. The reasons for this are not completely clear, although it is possible that they reflect a basic predisposition given the relative familiarity of real estate researchers with regression and time series methods, which provide a natural platform to understand spatial econometrics models. Regardless, in recent years there has been renewed interest in the application of geostatistical approaches, which recent research suggests can substantially improve the predictive accuracy of models (e.g. Case et al. 2004; Páez et al. 2008b). The third paper in the issue, by Tsutsumi and Seya, examines the applicability of spatial regression (lag and error autocorrelation models) and kriging for project evaluation purposes. One interesting aspect of this study is the discussion about the implications of the way spatial relationships are implemented in the various approaches. Use of spatial contiguity matrices in spatial lag and error autocorrelation models implicitly assumes that the spatial system is completely observed, whereas in kriging, the covariance matrix is modeled assuming that sites have been sampled. Past research on missing observations in zonal spatial systems includes the work of Griffith (1988) and more recently of LeSage and Pace (2004). Excluding the case of a purely autocorrelated model as implemented in Griffith (1988), other approaches necessitate explanatory variables at non-sampled sites, which requires that conditions at these sites be at least partially documented. Furthermore, these approaches require all sites that will be evaluated using the model to be identified a priori: new sites cannot simply be incorporated a posteriori, since their introduction would imply a different contiguity matrix than that used for estimation. The results of an empirical example that examines the impact of a new railway line connecting the cities of Tokyo and Tsukuba in Japan indicates that the estimated benefits are very different depending on the modeling approach adopted. It is currently unclear whether the ranking of benefits (higher for an ordinary least squares approach, lower for kriging) is somehow associated to the properties of each modeling approach or is a case specific finding. As Leonard and Murdoch illustrate, parameters can be biased if spatial effects are ignored, which supports the idea that the ordinary non-spatial model is an outlier. However, between spatial models, a more definitive answer to this question would necessitate an examination of the performance of the parameters in a simulation environment.

Two more papers in the issue are concerned with geostatistical applications in property assessment. Whereas the paper by Smith and Wu was concerned with data measurement in terms of the temporal intervals of observations, the second to last paper, by Yoo and Kiriakidis, is concerned with measurement of spatial information, which could be disaggregated for individual properties, or alternatively aggregated as area averages. A model, area-to-point kriging with external drift, or A2PKED, is proposed to deal with the situation where point and area measurements are available. This model is an extension of area-to-point kriging, and different from it in that A2PKED can accommodate additional covariates in the analysis. The model is demonstrated using a case study of Seoul, Korea, and a dataset partitioned 
into a training (or estimation) sample, and a validation sample. The results illustrate some desirable properties of A2PKED. Not only is this approach capable of using a potentially more common source of information (area averages), it also produces more accurate estimates of price compared to kriging and other non-spatial approaches. In addition, estimation in this approach is less affected by extreme observations, as the areal datum essentially functions as a check and balance for the influence of these observations. With respect to accuracy of predictions, a recent paper by Páez et al. (2008b) reports some important gains in performance when using moving windows approaches which are attributed to the segmentation of the market. However, these approaches are limited in their ability to use area variables, in particular if the areal support is large relative to the geographic market segments. In addition, moving window approaches require fairly large amounts of data, in order for local regressions to have a sufficient number of observations for estimation. On the other hand, A2PKED seems to perform well with smaller datasets, and naturally accommodates areal data. In fact, areal data appears to play the role of a proxy for local market conditions. These intriguing similarities suggest that a comparison of moving window approaches and A2PKED would be an interesting exercise to consider as a topic for future research.

The final paper in the theme issue, by Montero, Larraz, and Páez, also relates to the use of more common information to enhance assessment. This paper applies a different geostatistical approach, cokriging, to the study of commercial property prices using housing prices as ancillary variables. Housing prices are relatively easy to collect, and are frequently available for property assessment purposes. Data on commercial property prices, on the other hand, tend to be less common, partly because properties of this type are less numerous, but also because transactions in this market are less frequent, and so the market value of properties is characterized by more sparse observations. In order to improve the assessment of commercial property values, cokriging is proposed as an alternative to kriging. Cokriging operates on the same fundamental principles of kriging, making use of the additional information carried by spatial dependencies to refine predictions of values. This principle is the basis for spatial interpolation in both kriging and cokriging. Cokriging, in addition, is capable of using the spatial dependencies between two variables to improve the prediction of one of them. This approach is particularly appropriate when the ancillary variable is more common than the variable of direct interest, and when the two variables tend to covary. An application to the city of Toledo in Spain shows that cokriging, using an ancillary variable, can substantially improve predictive accuracy, thus offering an alternative to the usual problem of sparse data in the assessment of commercial property values.

Together, the five papers appearing in this issue are representative of the type of research currently being conducted in spatial hedonic analysis, as new problems and technical approaches come to the forefront of the research agenda. At the same time, the papers identify future research needs and point in the direction of some intriguing research possibilities, which already signify that spatial hedonic analysis will in all likelihood remain a vibrant and active arena for years to come. 


\section{References}

Baumont C (2009) Spatial effects of urban public policies on housing values. Pap Reg Sci 88(2):301-326

Bitter C, Mulligan GF, Dall'erba S (2007) Incorporating spatial variation in housing attribute prices: a comparison of geographically weighted regression and the spatial expansion method. J Geograph Syst 9(1):7-27

Bourassa SC, Hoesli M, Peng VS (2003) Do housing submarkets really matter? J Hous Econ 12(1):12-28

Can A (1992) Specification and estimation of hedonic housing price models. Reg Sci Urban Econ 22(3):453-474

Case B, Clapp J, Dubin R, Rodriguez M (2004) Modeling spatial and temporal house price patterns: a comparison of four models. J Real Estate Finance Econ 29(2):167-191

Chica-Olmo J (1995) Spatial estimation of housing prices and locational rents. Urban Stud 32(8):1331-1344

Clauretie TM, Daneshvary N (2009) Estimating the house foreclosure discount corrected for spatial price interdependence and endogeneity of marketing time. Real Estate Econ 37(1):43-67

Dubin RA (1992) Spatial autocorrelation and neighborhood quality. Reg Sci Urban Econ 22(3):433-452

Goodman AC, Thibodeau TG (2003) Housing market segmentation and hedonic prediction accuracy. J Hous Econ 12(3):181-201

Griffith DA (1988) Advanced spatial statistics: special topics in the exploration of quantitative spatial data series. Kluwer, Dordrecht

Harding JP, Rosenblatt E, Yao VW (2009) The contagion effect of foreclosed properties. J Urban Econ 66(3):164-178

Lesage JP, Pace RK (2004) Models for spatially dependent missing data. J Real Estate Finance Econ 29(2):233-254

Pace RK, Barry R, Clapp JM, Rodriquez M (1998) Spatiotemporal autoregressive models of neighborhood effects. J Real Estate Finance Econ 17(1):15-33

Páez A, Scott DM, Volz E (2008a) Weight matrices for social influence analysis: an investigation of measurement errors and their effect on model identification and estimation quality. Soc Netw 30(4):309-317

Páez A, Long F, Farber S (2008b) Moving window approaches for hedonic price estimation: an empirical comparison of modeling techniques. Urban Stud 45(8):1565-1581

Páez A, Le Gallo J, Buliung RN, Dall'Erba S (2009) Progress in spatial analysis: introduction. In: Páez A, Le Gallo J, Buliung RN, Dall'Erba S (eds) Progress in spatial analysis: methods and applications. Springer, Heidelberg, pp 1-13

Stetzer F (1982) Specifying weights in spatial forecasting models - the results of some experiments. Environ Plan A 14(5):571-584

Tse RYC (2002) Estimating neighbourhood effects in house prices: towards a new hedonic model approach. Urban Stud 39(7):1165-1180 